HUMANIDADES Y CIENCIAS SOCIALES

INVESTIGACIÓN

\title{
Revisitar la enseñanza de la lectura y la escritura mediada por tecnologías en educación superior
}

\author{
Clerici, Carolina
}

\section{Resumen}

Este artículo deriva de la tesis de maestría «La enseñanza de la lectura y la escritura mediada por tecnologías en la educación superior» de la Universidad Nacional de Córdoba, Argentina. Esa investigación buscó identificar, analizar y comprender propuestas de enseñanza de lectura y escritura que integran tecnologías en educación superior. El diseño combinó Teoría Fundamentada o Grounded Theory, método comparativo constante y muestreo teórico. En este artículo se incluyen los resultados relacionados con las recurrencias observadas en las propuestas de enseñanza de la lectura y la escritura mediada por tecnología y la decisión de revisitar la enseñanza a la luz del contexto actual.

Palabras clave: enseñanza de la lectura y la escritura; mediación tecnológica; educación superior

Este artículo deriva de la tesis de maestría «La enseñanza de la lectura y la escritura mediada por tecnologías en la educación superior» realizada en la Universidad Nacional de Córdoba, Argentina. La maestría fue llevada a cabo en contexto de la Beca de Cuarto Nivel de la UNER. Recibido el 16/1/2019 y aceptado el 2/3/2020.

DOI: https://doi.org/10.33255/3161/571

Autoría: Universidad Nacional de Entre Ríos (Argentina).

Contacto: carolina.clerici@uner.edu.ar 


\title{
Revisiting the teaching of reading and writing with ICT in higher education
}

\begin{abstract}
This article derives from the master's thesis «The teaching of reading and writing mediated by technology in higher education» of the National University of Cordoba, Argentina. This research sought to identify, analyze and understand teaching practices of reading and writing that integrate technologies in higher education. The design was Grounded Theory, constant comparative method and theoretical sampling. This article includes the results related to the recurrences observed in the teaching practices of reading and writing mediated by technology and the decision to revisit teaching in the light of the current context.
\end{abstract}

Keywords: teaching reading and writing; technological mediation; higher education

\section{Revisitar o ensino da leitura e da escrita mediado por tecnologias no ensino superior}

\section{Resumo}

Este artigo deriva da tese de mestrado $« O$ ensino da leitura e da escrita mediado por tecnologias no ensino superior» da Universidad Nacional de Córdoba, Argentina. Essa pesquisa buscou identificar, analisar e compreender propostas de ensino de leitura e escrita que integram tecnologias, na educação superior. O desenho combinou Teoria Fundamentada ou Grounded Theory, método comparativo constante e amostragem teórica. Este artigo inclui os resultados relacionados com as recorrências observadas nas propostas de ensino da leitura e da escrita mediada pela tecnologia e a decisão de revisitar o ensino à luz do contexto atual.

Palavras-chave: ensino da leitura e da escrita; mediação tecnológica; ensino superior 
Clerici, Carolina I Revisitar la enseñanza de la lectura y la escritura mediada por tecnologías en educación superior

\title{
1. Introducción
}

\author{
Las herramientas no llevan consigo la garantía de su éxito o fracaso, \\ del provecho o el daño que pueden provocar: todo depende del uso \\ que hagan de ellas las personas sensatas.
}

BURBULES Y CALLISTER, 2001: 27

Esta investigación fue realizada en el marco de la Maestría en Procesos Educativos Mediados por Tecnologías del Centro de Estudios Avanzados de la Universidad Nacional de Córdoba, Argentina. Tuvo como propósito identificar, analizar y comprender propuestas de enseñanza de lectura y escritura mediada por tecnologías o que integren tecnologías en educación superior con el objeto de construir generalizaciones que alienten la revisión de las prácticas de la enseñanza en dicho nivel. En el marco de la tesis mencionada, este artículo recupera algunas recurrencias en las prácticas de enseñanza de la lectura y la escritura mediada por tecnología en los relatos de los docentes entrevistados y la decisión de revisitar sus prácticas a la luz de las características del contexto actual.

A continuación, se incluye el marco de referencia desde el cual se abordó la enseñanza de la lectura y la escritura y el estado de la cuestión en relación con la mediación tecnológica en la educación superior.

\section{Marco de referencia}

\subsection{El impacto de las tecnologías en la cultura, la comunicación y el conocimiento}

Prácticamente no hay un solo ámbito de la vida humana que no se haya visto impactado por el desarrollo tecnológico. La omnipresencia de las TIC es al mismo tiempo una oportunidad y un desafío para la educación (OREALC/ UNESCO, 2013).

Vivimos en un nuevo escenario social: «la era de la posmodernidad», «la sociedad del conocimiento, «la era de la información», «la sociedad red», «la era digital», «la era de la conectividad» son algunas de las tantas denominaciones que proponen quienes inscriben sus estudios en el contexto contemporáneo. Se trata de un escenario de cambios en la concepción del tiempo, el espacio y el sujeto (Castells, 1999). En ese nuevo escenario, marcado por el ritmo acelerado, cobra otro sentido lo que entendemos por cercano y lejano, en el tiempo y el espacio.

El auge y la expansión de internet y de la industria tecnológica de la comunicación han permitido pensar en la idea de un acceso a la información 
de modo ilimitado, en cualquier tiempo y lugar. En ese escenario, las nuevas tecnologías atraviesan nuestra vida cotidiana, nuestros modos de pensar, de relacionarnos, de aprender, y la lectura y la escritura no están ajenas a esos atravesamientos.

Las nuevas tecnologías no solo constituyen un conjunto de herramientas, sino un entorno -un espacio, un ciberespacio- en el cual se producen las interacciones humanas. Cada vez más, la internet es un contexto en el cual se combinan y entrecruzan las actividades de indagación, comunicación, construcción y expresión. Cada vez más se describe a la Red como un «espacio público», un lugar donde la gente se reúne a debatir... No como un sucedáneo de la «interacción cara a cara», sino como algo distinto, de características singulares (Burbules y Callister, 2001: 18-19).

No se trata, entonces, de herramientas, sino de un entorno en el que suceden cosas. En este sentido, Buckingham (2008) se refiere a medios y no meras tecnologías. Reconoce que las tecnologías hacen posible la producción, circulación, consumo y uso. Sin embargo, se refiere a estos medios como formas de representación, cultura y comunicación, que están inmersos en procesos sociales y culturales. Cope y Kalantzis (2009) mencionan como características distintivas de los nuevos medios:

- Agencia: Los nuevos medios permiten escribir y crear. La distinción entre consumidor y productor se borra, el conocimiento y la autoridad se vuelven contingentes y provisorios.

- Divergencia: Esa misma agencia lleva a una gran diversificación e intensificación de las diferencias individuales.

- Multimodalidad: El cambio del caracter por el pixel hace posible la combinación de diversos modos de representación en un mismo «texto».

- Conceptualización: El paso del consumo pasivo a la posibilidad de producción conlleva la necesidad de conocer la arquitectura técnica y social, la mecánica de la comunicación.

Estas características de los nuevos medios implican un gran desafío para la educación ya que ya no se trata de preparar para la recepción de información y el consumo pasivo, sino que surgen alfabetizaciones múltiples que es necesario atender. Cope y Kalantzis (2009) las describen en relación con las cuatro dimensiones mencionadas:

- Diseño: La institución educativa debe transformarse en una comunidad de producción de conocimiento y es esencial que los alumnos tomen conciencia de esa posibilidad. 
- Estilos de aprendizaje: La gran divergencia hace imposible que todos aprendan al mismo tiempo y en la misma página del libro, tal como en la escuela tradicional. Esto requiere atención especial a la hora de la evaluación, entendida no como medición de saber sino como acompañamiento y retroalimentación del aprendiz.

- Sinestesia: Al igual que Buckingham (2008), estos autores insisten en que los nuevos medios no pueden aportar desde una visión de fragmentación en asignaturas ni desde una pedagogía que sobrevalora un modo de representación, como es la escritura. En cambio, es necesario pensar modos múltiples de representación que atiendan a los diferentes estilos de aprendizaje y representación de los aprendices. Si la palabra no se entiende, entonces una imagen podrá ser de utilidad y hacer más clara la escritura.

- Metacognición: Los nuevos medios requieren conceptualización y metacognición al involucrar al alumno como co-constructor de conceptos, definidor, hacedor de teoría, crítico y analista.

Tal como expresa Buckingham, estamos frente a:

la proliferación de emisores; la individualización del acceso a los medios; el advenimiento de los llamados medios interactivos; el creciente potencial de utilización de los medios para la comunicación y la participación; y la constante comercialización de los medios (2006: 3).

Estamos inmersos en una cultura participativa (Jenkins, 2009) y de la conectividad, una nueva fase de la socialidad en línea, en la cual las tecnologías y los usuarios co-evolucionan, aunque definitivamente los medios conectivos avanzan cada vez más sobre las relaciones humanas, codificándolas como datos y convirtiéndolas en productos con valor comercial (Van Dijck, 2013, 2016).

En ese contexto, cobra importancia el desarrollo de habilidades como encontrar, organizar y utilizar información:

Saber cuándo, dónde y cómo buscar información, ser capaz de seleccionarla en función de unos objetivos, procesarla (leerla) y elaborarla adecuadamente para después utilizarla (escribirla, exponerla, aplicarla) con el fin de resolver un determinado problema constituyen cadenas de competencia de urgente instauración curricular (Monereo y Pozo, 2007: 17).

En el entorno digital se valoran las habilidades críticas para poder usar esta información de modo inequívoco (Burbules y Callister, 2001; Cassany, 2002). Y no solo se trata de leer críticamente los medios, sino de escribir en 
ellos, sostiene Buckingham (2006). Para este autor, la ventaja de las nuevas tecnologías está en la posibilidad de que los estudiantes produzcan y compartan sus propios medios. Es por eso que se habla de una arquitectura de la participación. Los recursos que ofrece un hipertexto potencian la creatividad y significación. Y, al mismo tiempo, nos exige desempeñar un papel mucho más activo durante la lectura: aprender a explorar las opciones que ofrecen internet y las TIC. Estamos en presencia de nuevas relaciones con el conocimiento y nuevos usos. Hoy, estamos llamados a establecer vínculos más horizontales, participar y pertenecer de un modo nuevo. La sociedad actual requiere, además, el desarrollo de competencias como interactuar a través de medios electrónicos; comprender y producir géneros nuevos; en resumen, demanda el desarrollo de la competencia comunicativa multimodal (Cassany, 2002).

Se trata de desarrollar habilidades de lectura crítica y de participación. Las nuevas tecnologías impactan en las prácticas culturales y de comunicación al generar la posibilidad de «intervención, reescritura, modificación y cambio de sentido de los productos ya existentes que, de alguna manera, están ahora abiertos a la resignificación» (Dussel y Quevedo, 2010: 23). Es posible observar que lo que se da como hecho puede ser modificado, reescrito, transformado, y que cada uno de nosotros puede ser parte de ese cambio.

La interactividad hace referencia al modo de relacionarnos con otros y construir colaborativamente, es un proceso social de intercambio y de construcción colectiva del saber. La nueva sociedad requiere del sujeto determinadas competencias para conocer, para vincularse y para poder ingresar a la lógica del mercado y la participación.

Se plantean así vínculos más horizontales, que nos recuerdan permanentemente que estamos llamados a crear, actuar e intervenir. Hay quienes ven en la tecnología una fuerza liberadora que permite formas autónomas de comunicación y comunidad. Otros, en cambio, comparten su preocupación por las nuevas lógicas de expulsión que se potencian con las tecnologías (Sassen, 2014), no solo en términos de falta de recursos materiales -computadoras, conexión a internet- sino también en términos de falta recursos simbólicos -como son la capacidad de participación en esos entornos a través de la lectura y la escritura-.

El impacto que han tenido las tecnologías en la cultura, la sociedad y el conocimiento interpela a las instituciones educativas. Esto nos obliga a reflexionar «sobre las formas en que podemos crear deliberadamente experiencias y oportunidades de aprendizaje que amplíen el ámbito de las posibilidades humanas» (Burbules y Callister, 2001: 71) sin dejar de preocuparnos por quienes quedan al margen de estas experiencias y oportunidades. 


\title{
2.2. Leer y escribir en la cultura digital
}

Sabemos que el entorno digital impacta en las prácticas comunicativas; leer y escribir cambian, surgen nuevos géneros discursivos, nuevos roles, y todo esto significa un gran desafío para la educación, especialmente para la alfabetización académica. La incorporación de las nuevas tecnologías no implica per se el desarrollo de buenas propuestas de enseñanza. Ante esta realidad, una pedagogía adecuada al contexto actual, una ciberpedagogía (Fischer, 2005) o una pedagogía del ciberespacio (Apolo, Bayés y Hermann, 2016), debe tener en cuenta los nuevos modos de configurar la realidad.

\begin{abstract}
el valor de la tecnología digital (...) depende en gran medida de las relaciones pedagógicas que se establezcan alrededor de ella, por ejemplo, de cómo se habilita el acceso de los alumnos a las habilidades y competencias que necesitan, en qué medida se les permite controlar el proceso y en qué medida pueden dialogar con sus docentes y pares. También depende, en términos más amplios, de los contextos sociales en los que se utiliza: de las motivaciones de los estudiantes, de las maneras en las que la producción cultural se relaciona con otros aspectos de su vida, del público para sus producciones, etcétera. (Buckingham, 2008: 219)
\end{abstract}

Ahora bien, los nuevos medios de comunicación pueden perpetuar la forma de enseñanza clásica basada en la transmisión, y de hecho hay estudios que muestran que mucho de la inclusión de las TIC en el aula ha tratado de hacer lo mismo que antes, pero con nuevas tecnologías o que la inclusión de la tecnología no agrega valor pedagógico (Jaramillo Marín y Ruiz Quiroga, 2009).

La centralidad de las propuestas educativas -que observamos en su generalidad-, en su admiración por lo tecnológico, ha provocado propuestas focalizadas en la implementación del recurso como una manera de reproducir prácticas educativas tradicionales «maquilladas». Es decir, se sobrevalora lo tecnológico por sobre lo pedagógico.

Estos nuevos modos de leer, escribir y aprender con tecnología obligan a los docentes a replantearse sus prácticas de enseñanza, recuperar el sentido fundante de la educación mediada por tecnologías, desnaturalizar la mirada a partir de la reflexión. En lugar del sistema tradicional centrado en horas de clase presencial, este nuevo sistema se centra en el trabajo realizado por los estudiantes. En este sentido, las TIC se presentan como una oportunidad para gestionar de forma adecuada el trabajo con ellos. Esto nos lleva a dos interrogantes: ¿Por qué un docente decide incluir la tecnología en el aula para la enseñanza en general? ¿Y por qué decide incluirla en la enseñanza de la lectura y la escritura en particular? 
Para el abordaje de las razones por las que los profesores pueden verse impulsados a incluir la tecnología en el aula, Litwin (2008) ofrece dos perspectivas o escenarios: la tecnología para ayudar a la comprensión y la tecnología para generar interrogantes de nuevo tipo. En el primer escenario, la autora incluye la introducción de las tecnologías porque favorecen la comprensión, porque están inmersas en las prácticas profesionales, porque proveen información actualizada o porque permiten desarrollar trabajo en equipo. En palabras de la autora, «se trata de creaciones tecnológicas para proveer de contenidos, ayudar a pensar, ayudar a pensar bien y con el otro» (Litwin, 2008: 168).

Para pensar el uso de la tecnología en el aula, Litwin (2008) retoma algunos interrogantes de Meirieu: ¿Qué finalidades se persiguen realmente a través de su uso? ¿Las herramientas propuestas sirven realmente para las finalidades que quiero conseguir y es posible que los conocimientos movilizados puedan iluminar mi actividad? (Meirieu, 2001: 111-112, citado en Litwin, 2008). La potencia de la tecnología radica en ofrecer una posibilidad para el desarrollo de la mente, las «que son invitaciones a pensar, las que rompen con las tradiciones, con el canon y establecen nuevas maneras de analizar y profundizar en un tema» (Litwin, 2008: 172).

Y también existen otras razones para incluir las tecnologías. Kap (2014) nos recuerda que hay un fuerte componente afectivo en la utilización o no de las nuevas tecnologías donde se ponen en juego dimensiones muy complejas que tienen que ver con el poder, con el lugar del saber. La autora argumenta que los docentes que están a favor de las nuevas tecnologías se manifiestan apasionados, comprometidos, intrigados, curiosos, y las toman como un desafío. Reconoce que sienten incertidumbre, que están corriendo un riesgo muy grande pero que están dispuestos a enfrentarlo. Son esos docentes «conmovidos por las tecnologías» que buscan su inclusión con sentido pedagógico y didáctico potente.

Si bien las tecnologías han generado cambios trascendentales que han impulsado la modernización de programas educativos, el quehacer didáctico no ha logrado una adecuación genuina a los soportes y dispositivos digitales ni ha logrado desarrollar estrategias de cara a las demandas y desafíos de una sociedad globalizada, tecnologizada y digitalizada (Arnao Vásquez y Gamonal Torres, 2016; Jarvio, 2011) y en un contexto que se caracteriza por la complejidad, la velocidad y la imprevisibilidad (Lion, 2012).

En cuanto al grado de penetración de la tecnología en la enseñanza, Maggio (2012) distingue dos tipos: inclusiones efectivas, en las que la incorporación de las tecnologías no responde a motivos propios de los docentes, 
Clerici, Carolina I Revisitar la enseñanza de la lectura y la escritura mediada por tecnologías en educación superior

el docente las incluye pero no reconoce su valor para la enseñanza ni las integra con sentido didáctico; y la inclusión genuina, en la que el uso de la tecnología depende de la importancia que le asigna el docente, y este reconoce su valor en los campos de conocimiento disciplinar objeto de la enseñanza.

Hoy es necesario reconocer que nuestras mentes, y en especial las de los niños y jóvenes, están atravesadas por entornos tecnológicos que soportan comunicaciones, relaciones, consumos culturales y entretenimientos. Cuando no hacemos este reconocimiento, se genera un vacío entre los modos como nuestros alumnos conocen y pueden aprender y nuestras propuestas para favorecer que ello ocurra. Este vacío es cognitivo, cuando no reconocemos el carácter de los procesos que tienen lugar efectivamente; es cultural, cuando no entendemos a nuestros alumnos como sujetos y miembros de culturas particulares; y, finalmente, es pedagógico, cuando construimos y llevamos adelante propuestas que en lugar de suscitar lazos con esos sujetos sociales, cognitivos y culturales, caen en el vacío propio de nuestra falta de reconocimiento de sus modos de conocer, relacionarse, interactuar y, por ende, aprender. Al tiempo constatamos como fenómeno duro, aunque casi lógico, que nuestros alumnos no aprenden como quisiéramos. (Maggio, 2012: 22)

Maggio propone encontrarle a la tecnología un sentido pedagógico y didáctico potente, nos invita a generar propuestas didácticas que alienten a nuestros alumnos a aprender de modos enriquecidos y valiosos.

\subsection{La enseñanza de la lectura y la escritura mediada por tecnología como objeto de estudio: el estado de la cuestión}

Las investigaciones sobre la enseñanza de la lectura y la escritura en la educación superior en América Latina han aumentado considerablemente en las últimas décadas (Navarro y otros, 2016). Estos estudios han contribuido a la delimitación de un espacio disciplinar, que puede llamarse alfabetización académica, análisis del discurso académico, didáctica de la lectura y la escritura, entre otras denominaciones utilizadas en la literatura especializada.

Navarro y otros (2016) ofrecen una síntesis de los antecedentes más relevantes sobre la temática. Uno de los principales aportes se sitúa en 1980 en Argentina, con el lanzamiento de la revista Lectura y Vida. Revista Latinoamericana de Lectura. En 1987, desde la cátedra Semiología de la Universidad de Buenos Aires, se llevó a cabo un proyecto pionero de investigación-acción para la enseñanza de la lectura y la escritura en la educación superior, que contribuyó a la institucionalización de talleres de lectura y escritura para 
ingresantes al nivel superior. A mediados de los 90, la creación de la Cátedra UNESCO para el Mejoramiento de la Calidad y Equidad de la Educación en América Latina con base en la Lectura y Escritura marcó el inicio del campo disciplinar e impulsó el intercambio y la sistematización en América Latina.

Desde entonces se han publicado estudios que aportan una mirada reflexiva sobre la alfabetización académica (Carlino, 2003; Cassany y Morales, 2008; Castelló, 2007; Estienne, 2006; Parodi y Burdiles, 2015). No obstante, los estudios sistemáticos de este campo disciplinar en prácticas mediadas por tecnología en la educación superior continúa siendo escaso.

Entre los estudios publicados que dan cuenta de estas preocupaciones, particularmente del uso de la tecnología en propuestas de enseñanza de la lectura y la escritura, es posible observar que la lectura ha recibido mayor atención por parte de los investigadores que la escritura (Gallego Ortega, García Guzmán y Rodríguez Fuente, 2013), a diferencia de lo que sucede en estudios en los que no se aborda la relación explícita con la tecnología (Navarro y otros, 2016). En ambos casos predominan las investigaciones realizadas en niveles educativos primario y secundario, con grupos específicos como niños con dificultades de aprendizaje o aquellas que se han dedicado a aspectos parciales del proceso de escritura como la planificación o la revisión.

Entre los estudios que abordan la lectura mediada por tecnología en el ámbito específico de la educación superior, abundan aquellos centrados en el impacto de las tecnologías en los modos de leer y en las prácticas de lectura de los jóvenes universitarios. A partir de estos se puede mencionar la diferencia entre la lectura de textos impresos y digitales (Romo González y Villalobos Alonzo, 2009); la incidencia de la lectura hipermedial en la comprensión lectora (Trillos-Pacheco, 2013), y el uso de las TIC como motivación e incentivo para los estudiantes y como estrategia didáctica para contribuir al aumento de la comprensión lectora (De Castro y otros, 2014, 2015).

Entre los estudios que han abordado la escritura mediada por tecnología en el ámbito específico de la educación superior, se han publicado investigaciones y relatos de experiencias de uso de diferentes tecnologías, recursos y aplicaciones para la escritura. Tal es el caso del uso de esquemas digitales de escritura, que son estructuras modelo básicas que sirven de andamiaje y orientación al estudiante en su proceso de producción (Figueroa, Aillon y Fuentealba, 2014; Fuentealba, Figueroa y Aillon, 2011). También se destacan estudios sobre el uso de blogs para mejorar la escritura (Álvarez y Bassa, 2013). En esta misma línea se encuentra la escritura de ensayos académicos colaborativos (Figueroa y Aillon, 2015), por mencionar algunos. 
Finalmente, entre los estudios en los que se hace presente la tecnología en relación con la lectura y la escritura son frecuentes los escritos teóricos. Entre ellos, reflexiones sobre nuevos modos de circulación de la palabra mediada por tecnología (Kap, 2016), y sobre la necesidad de contar con un modelo integrador de alfabetización académica y digital (López-Andrada, 2016). Además de estudios teóricos, muchas publicaciones versan principalmente sobre la utilización de entornos específicos como redes sociales, plataformas virtuales institucionales y blogs para la escritura, en especial desde el enfoque del estudiante, de los aprendizajes desarrollados y de la valoración que hacen de la experiencia. Reale (2008) ha destacado una serie de aspectos positivos para el desarrollo de las habilidades de lectura y escritura a través del uso de blogs. Álvarez, García y Qués (2010) han señalado que la convergencia de medios, la hipertextualidad y la interactividad propias de las nuevas tecnologías ofrecen posibilidades de autogestión y colaboración del trabajo que pueden influir positivamente en la comprensión y producción de textos. Álvarez y Taboada (2016) han realizado experiencias de uso de Facebook y Moodle con énfasis en la reformulación.

La enseñanza de la lectura y la escritura mediada por tecnología en la educación superior es un terreno en el que son escasas las publicaciones de estudios empíricos, es decir que existe un espacio de indagación de la problemática. En un estudio comparativo de los temas de investigación más recurrentes en materia de lectura y escritura, Navarro y otros (2016) muestran que los temas sobre los que más se ha publicado en la región son el discurso, la lectura y la escritura estudiantiles y la enseñanza, dejando en segundo plano la lectura y la escritura en relación con la investigación y los géneros discursivos, y en el último lugar, los artículos que indagan la relación con ambientes virtuales. En esta clasificación por subtemas de investigación no se menciona la mediación tecnológica en general, más allá de los entornos virtuales. Además, son escasos los estudios en América Latina y desde un enfoque centrado en las propuestas de los docentes, desde sus criterios pedagógicos, las decisiones por las que deciden proponer la lectura y la escritura mediada por tecnología en la educación superior. En esta carencia y necesidad se sustentó la presente investigación.

\section{Diseño metodológico}

En el trabajo de investigación en el que se enmarca este artículo se buscó identificar, caracterizar, analizar y comprender las propuestas de enseñanza de la lectura y la escritura mediada por tecnologías en la educación superior tercia- 
ria y universitaria, los factores que facilitan u obstaculizan la puesta en marcha de dichas prácticas y los criterios pedagógicos con los que se plantean. Se buscó también aportar a la teoría acerca de las prácticas de la enseñanza a partir de sus derivaciones para pensar estos procesos en el ámbito de la educación superior terciaria y universitaria, recuperándose la centralidad de la mirada de los docentes. En este sentido, interesó particularmente indagar las decisiones de los docentes, sus fundamentos para usar las nuevas tecnologías y el sentido pedagógico y didáctico que le han encontrado a lo que hacen. Se trabajó desde un enfoque epistemológico interpretativo, de la didáctica crítica, que buscó la construcción teórica a partir de prácticas reales con el propósito de mirar lo que sucede en la alfabetización académica.

Se propuso un estudio desde una lógica inductiva de generación de categorías de análisis que permitiesen conocer el fenómeno bajo estudio desde la descripción de prácticas concretas. El trabajo no se desarrolló en etapas, sino que se fue construyendo en torno a momentos críticos en los que el trabajo de campo impactó en la revisión de los supuestos y la generación de nuevas preguntas de investigación.

Para ello se utilizó el diseño de Teoría Fundamentada o Grounded Theory (Glaser y Strauss, 1967; Strauss y Corbin, 2002). Se trata de un diseño que analiza inductivamente la información empírica y genera categorías teóricas. En consonancia con ello, se trabajó con el método comparativo constante y el muestreo teórico. Esto implicó que a medida que se avanzaba en el trabajo de campo se codificó y analizó la información empírica simultáneamente y se desarrollaron conceptos a partir de la comparación continua entre la información empírica y la teoría en una relación dialéctica; también implicó que se seleccionaran casos o informantes en la medida que fueron agregando nueva información para la comprensión del fenómeno bajo estudio.

Se realizaron entrevistas a profesores que llevan adelante propuestas de enseñanza de la lectura y la escritura con tecnologías en la educación superior. Se decidió trabajar con una muestra intencional inicial de cuatro docentes que tienen a cargo los talleres de Oralidad, Lectura, Escritura y TIC de las carreras presenciales en una institución terciaria de la ciudad de Gualeguaychú, Argentina. Esta decisión responde a que se trata de espacios en los que explícitamente se abordan la lectura y la escritura mediadas por tecnologías en el ingreso a la educación superior. Se trata de una muestra de casos-tipo y de expertos. Durante las primeras entrevistas a estos docentes se contempló ampliar la muestra a docentes que se desempeñan en áreas afines en el nivel superior, en carreras presenciales y semipresenciales, incluyendo universidades en otras localidades de Argentina. Finalmente, se contactaron 
casos en el resto de América Latina para tener una visión más global de lo que se realiza en términos de lectura y escritura mediadas por tecnología. La muestra final quedó compuesta por catorce docentes universitarios que se desempeñan en el contexto del ingreso a la educación superior (ver Figura 1). Los docentes entrevistados tienen formación pedagógica de algún tipo, muchos con estudios de posgrado orientados a la educación, y años de experiencia docente. Dan cuenta de una trayectoria de actualización en la integración de TIC y, en muchos de los casos, con el acompañamiento de la investigación y la sistematización de experiencias.

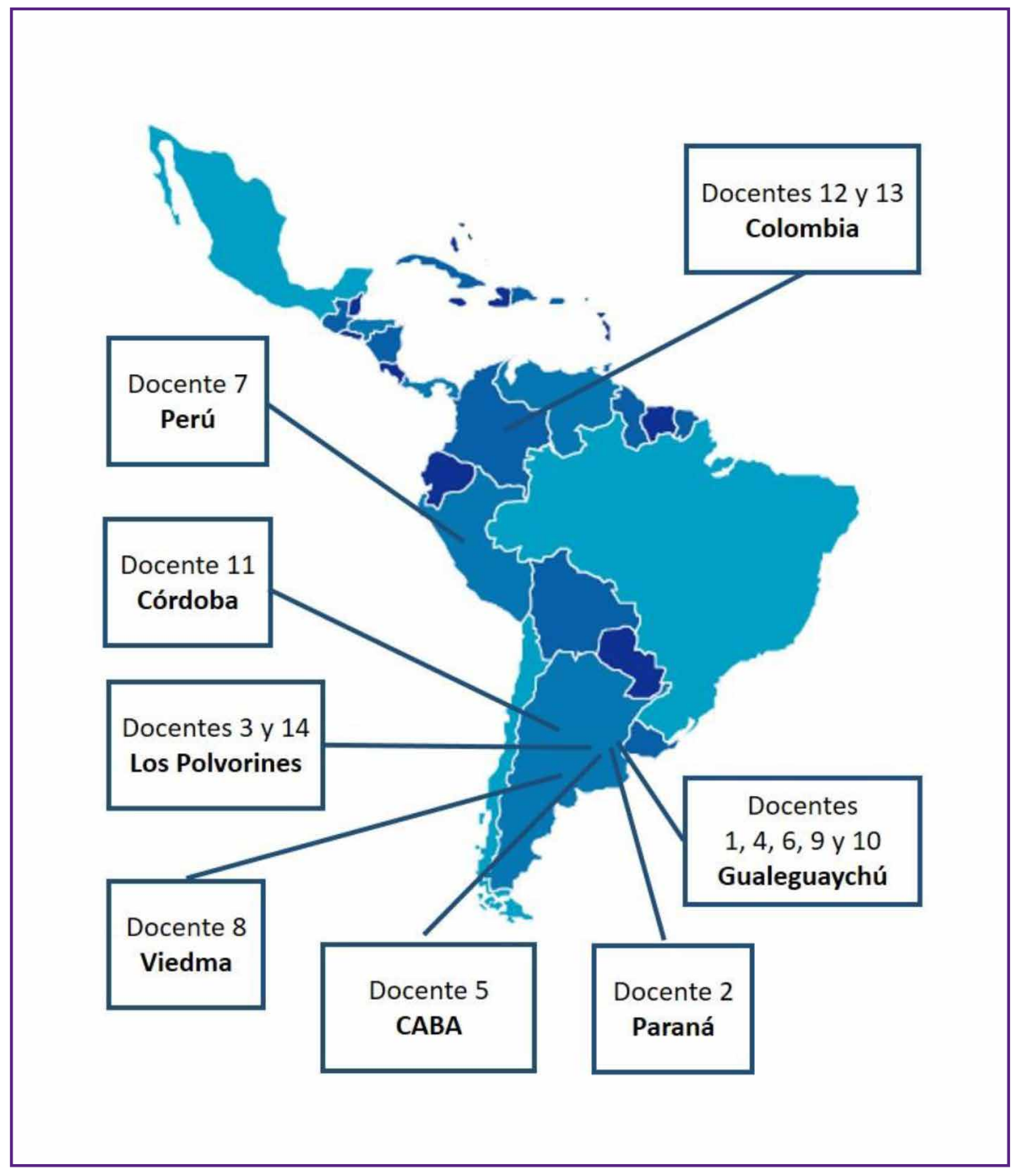

Figura 1. Mapa ilustrativo de la muestra entrevistada

Fuente: elaboración propia. 
La entrevista fue semiestructurada y flexible. Se incluyeron interrogantes que buscaron conocer las percepciones de los profesores en relación con el rol de las tecnologías en la enseñanza de la lectura y la escritura, las actividades concretas que han realizado y la valoración de las mismas desde su propia perspectiva, los factores que han facilitado u obstaculizado la implementación de estas propuestas, y las posibilidades de trabajo que se abren con la inclusión de la tecnología. Como es propio en la investigación cualitativa desde una lógica inductiva, el instrumento se fue adaptando a medida que se avanzó en la obtención de información y su interpretación.

La decisión de trabajar con entrevista responde a que es una de las instancias más apropiadas para acceder al universo de significaciones de los sujetos, y permite conocer referencias a acciones presentes y pasadas que no son accesibles para el investigador (Guber, 1991). En este sentido, y en línea con lo propuesto por Taylor y Bogdan (1987), para la entrevista en profundidad se hizo hincapié en la búsqueda de los significados, perspectivas y definiciones de los docentes entrevistados y en el modo en que ellos ven, califican y experimentan la enseñanza de la lectura y la escritura mediada por tecnología.

Además de las entrevistas, en algunos casos fue posible observar documentos y materiales utilizados por los docentes en el contexto de las propuestas que describieron. Entre estos documentos se encuentran consignas de trabajo, guías de lectura o escritura, producciones de los estudiantes y retroalimentación ofrecida por el docente, por mencionar algunos. Esto supuso un trabajo de análisis documental que se vio enriquecido por el acceso a algunos entornos de trabajo como plataforma la Moodle institucional, grupos en Facebook y documentos en Google Drive. Esta información empírica complementaria permitió reconstruir información de contexto.

El procesamiento de la información se realizó a través del método comparativo constante (Glaser y Strauss, 1967), que consiste fundamentalmente en comparar las conceptualizaciones que surgen de la información empírica. Se utilizó una matriz de tres columnas (evento o incidente, comentarios del entrevistador y categorías analíticas), se construyeron memos con las categorías emergentes durante la codificación abierta y se seleccionaron categorías centrales durante la codificación axial. Finalmente se produjo la escritura de la teoría en la codificación selectiva, acompañada de herramientas típicas de análisis cualitativo como esquemas, mapas conceptuales y matrices. Como es propio del método comparativo constante, las categorías que se construyeron a partir de la información empírica se combinaron con la incorporación de otras elaboradas en investigaciones previas cuando resultó pertinente (Goetz y Le Compte, 1988). 


\section{Resultados y análisis}

En este artículo se presentan las recurrencias y persistencias en los relatos de los docentes entrevistados. Estos resultados se centran particularmente en el modo en que los docentes integran la tecnología en sus propuestas, así como las principales dificultades que encuentran en sus estudiantes y cómo aprovechan el potencial de las TIC para hacer frente a estas.

\subsection{Algunas recurrencias observadas}

Si bien los entrevistados relataron experiencias muy diferentes y con objetivos también diversos, fue posible registrar recurrencias, aspectos en común y persistencias en los modos en que llevan adelante sus propuestas de enseñanza de lectura y escritura mediada por tecnología en la educación superior.

Los entrevistados comparten el interés por la tecnología y el uso en su vida cotidiana principalmente para cuestiones ligadas a la comunicación personal y a la preparación de las clases, al punto que muchos expresaron que no imaginan la vida ni la enseñanza sin tecnología. Muchos han sido estudiantes en cursos virtuales y semipresenciales y expresan que han podido transferir esos aprendizajes a sus clases como docentes. Para ellos, la posibilidad de pensar actividades de lectura y escritura con TIC es una consecuencia directa de su trayecto de formación, además de su interés personal en la tecnología.

Por otra parte, consideran que la tecnología debe incluirse en la educación en general porque posibilita la participación. Muchos de los entrevistados remarcaron el hecho de que a través de las TIC «aparecen cosas que en la clase no se ven». Esto fue mencionado en relación con estudiantes que no suelen participar en las clases presenciales, pero que «se animan» a realizar aportes en los entornos virtuales, se animan a participar sin inhibiciones. También se mencionó esta posibilidad de descubrir aspectos desconocidos de los estudiantes en casos en que aquellos que no se destacan en la presencialidad comparten producciones creativas y de alta calidad cuando se trata de tareas a realizar en entornos virtuales o redes sociales. Finalmente, la participación también fue asociada a «una participación que uno no ve», es decir, la de aquellos que leen y no escriben.

Otro motivo compartido fue el interés que provocan las tecnologías en los estudiantes. Los entrevistados consideran que las actividades propuestas en redes sociales o desde una intención lúdica y competitiva generan en los estudiantes deseos de participar, de leer y escribir, «les dan ganas de hacer». Los docentes coinciden en que las TIC contribuyen a que los estudiantes participen y lo hagan con gusto. 
En cuanto a la inclusión de la tecnología para la enseñanza de la lectura y la escritura en particular, los entrevistados expusieron muchas razones para justificarla. Es posible sintetizar estas razones en tres principales: el aprovechamiento del tiempo, la posibilidad de observación del proceso de aprendizaje y la oportunidad de hacer frente a las dificultades con las que se encuentran los estudiantes que ingresan a la educación superior.

Asimismo, destacaron la posibilidad de contar con tiempo fuera de la clase para repasar y ejercitar. En este sentido se concibe el aprendizaje como algo que puede adaptarse y flexibilizarse a las necesidades y los ritmos del aprendiente y provisto «justo a tiempo» (Buckingham, 2008: 42).

Otro aspecto que destacaron es la posibilidad de ver «lo que en la clase no se ve». Una de las entrevistadas (Docente 11) comentó una experiencia en la que trabajó comparativamente una actividad de escritura colaborativa en la clase presencial y en un documento de Drive. Ella notó que los estudiantes se desempeñaban de manera diferente en la clase presencial y en el documento colaborativo, que la presencia de las voces de todos se dio con más fuerza en el trabajo en línea. Tal como lo expresó:

La forma de enunciación cambia, en el sentido de que los textos que son escritos en el grupo de manera presencial tienen generalmente una sola voz, digamos hay uno que ocuparía el lugar de líder, la voz autorizada, recibe los aportes de los demás y entonces, por ejemplo, las personas más tímidas aportan muy poco o siempre se sienten condicionadas... o no hablan directamente. Mientras que en el texto digamos mediado tecnológicamente se animan más a intervenir, a decir... Justo anoche estaba leyendo una chica que dice «no, bueno, no, esto no», que nunca lo escuché en el grupo presencial. Y creo que eso va siendo un texto más polifónico, no enunciado por uno solo. (Docente 11)

Otro de los profesores (Docente 6), al referirse a una actividad de escritura de microficción en Twitter, afirmó que aparecen cosas que en el aula no se ven, que se pueden observar modos de escribir y participar de los estudiantes que no se hacen visibles en la clase presencial. Así lo expresó:

Creo que fue interesante porque empiezan a aparecer cosas que tal vez en el aula uno no ve en ciertos alumnos, y cuando tienen tiempo como para producir algo resultan cosas muy interesantes. (Docente 6)

La tecnología como oportunidad para hacer frente a las dificultades con las que se encuentran los estudiantes fue otra regularidad entre los testimonios de 
los entrevistados. Destacaron la posibilidad que abren las tecnologías de escribir textos no convencionales, de contextualizar la información, de visualizar el problema retórico en torno a la comunicación, de acompañar la escritura como proceso, de ejercitar y practicar más allá del aula, de generar intercambio y discusión en torno a la tarea y, con ello, mayor reflexión, apropiación más profunda y toma de conciencia de los procesos de lectura y escritura.

Entre las prácticas de lectura con tecnologías descriptas por los entrevistados predominan las actividades de búsqueda, selección y procesamiento de información que luego se plasman en actividades de síntesis, transformación y presentación oral o publicación en variados formatos, como presentaciones digitales, mapas conceptuales y blogs. Algunos entrevistados mencionaron actividades asociadas al enriquecimiento de la lectura a partir de recursos multimediales -antes, durante y después de la lectura-, entre los que mencionaron videos, pinturas, poemas, otros textos, textos de apoyo producidos por el propio docente y otros a los que los estudiantes acceden por iniciativa propia para informarse.

En cuanto a las prácticas de escritura, han mencionado la elaboración de infografías como textos multimediales; escritura de microficción en Twitter; elaboración de relatos colectivos, escritura creativa y narrativa breve en Facebook; reformulación de fragmentos en Facebook; escritura colaborativa en Google Docs; elaboración de portafolios digitales en blog y en Drive; publicación de textos y consignas escritas en blogs; escritura y publicación de cuentos digitales, entre otras.

Los testimonios de los entrevistados dan cuenta de propuestas que abordan la lectura y la escritura en un ciclo continuo lectura-escritura, un proceso complejo y extenso en el marco del cual se piensa una serie de actividades en las que los estudiantes leen y escriben y vuelven a leer y escribir con el foco puesto en el conocimiento de diferentes tipos de textos, el desarrollo de habilidades de lectura y escritura o el aprendizaje de contenidos específicos. En la Figura 2 se ofrece una síntesis, esquemática, de puntos en común en muchas de las propuestas analizadas en esta investigación. Se observa la centralidad del texto con el que se trabaja y, en torno a este, una serie de actividades de búsqueda de información, análisis y comprensión, comunicación y socialización. 


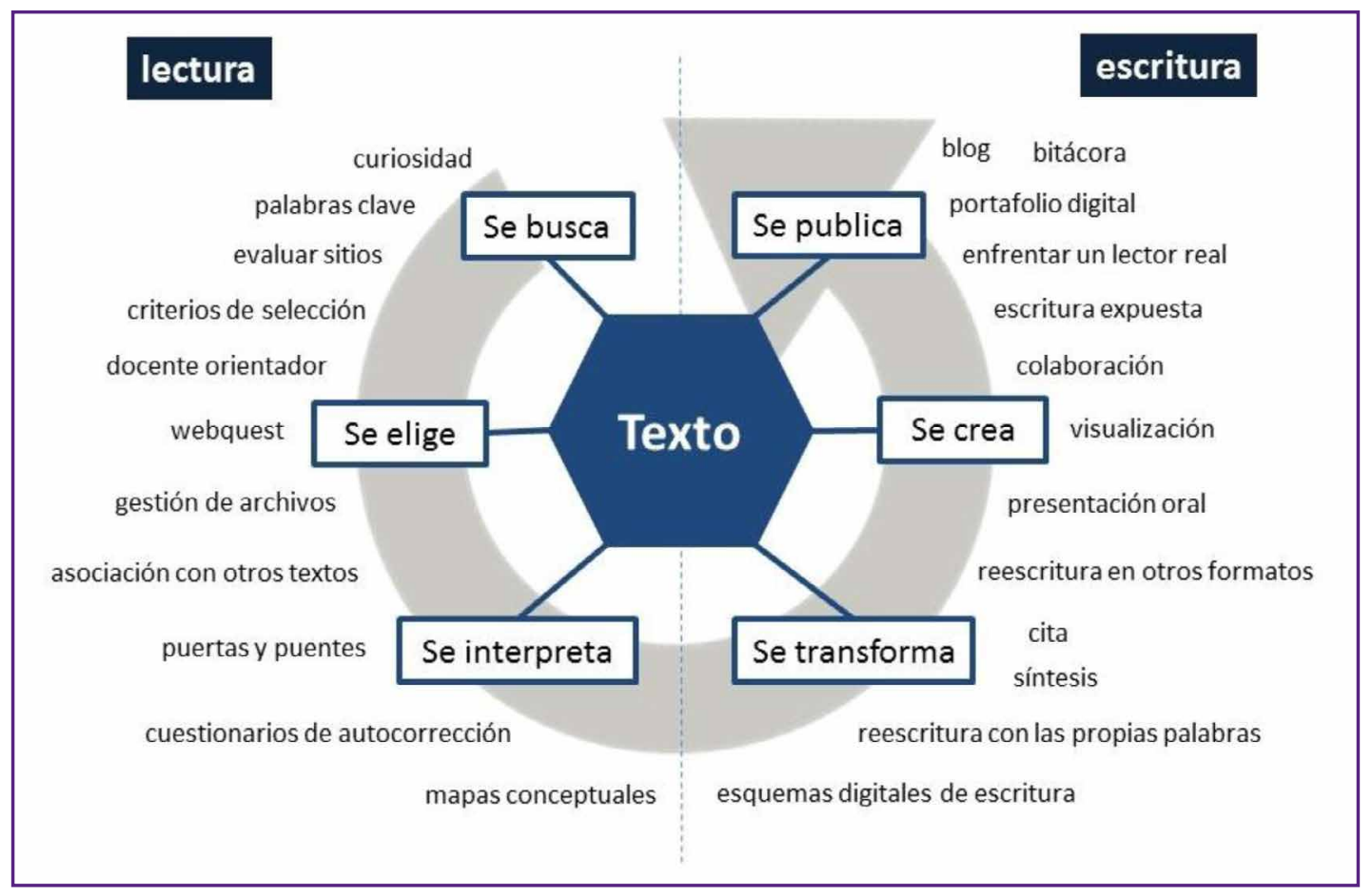

Figura 2. Esquema del ciclo continuo lectura-escritura mediado por tecnología

Fuente: elaboración propia.

Entre las tecnologías que utilizan con más frecuencia, en este ciclo continuo lectura-escritura, se destaca el uso de Facebook por sobre las demás redes sociales y herramientas. Sus argumentos se apoyan en la gran popularidad y facilidad de acceso de dicha red, lo que $\operatorname{Van} \operatorname{Dijck}(2013,2016)$ confirma al describir a Facebook como el mayor gigante, la red social más extendida y la que ofrece las mejores posibilidades de comunicación de masas. La Tabla 1 incluye las aplicaciones y recursos más usados por estos entrevistados y los motivos por los que han decidido incluirlos en sus propuestas de enseñanza de lectura y escritura. 
Clerici, Carolina I Revisitar la enseñanza de la lectura y la escritura mediada por tecnologías en educación superior

Tabla 1. Las tecnologías más usadas por los entrevistados para actividades de lectura y escritura y razones para su uso

\begin{tabular}{|c|c|}
\hline \multirow[t]{2}{*}{ Algunas tecnologías usadas... } & Algunas razones para usarlas... \\
\hline & - Es popular y de fácil acceso. \\
\hline \multirow{2}{*}{ ffacebook. } & - Permite la interacción en tiempo real. \\
\hline & $\begin{array}{l}\text { - Permite la generación de grupos, sin la necesidad de ser } \\
\text { contacto. }\end{array}$ \\
\hline \multirow{2}{*}{$\triangle$ Google Drive } & $\begin{array}{l}\text { - Permite la interacción para la producción de un texto } \\
\text { conjunto. }\end{array}$ \\
\hline & $\begin{array}{l}\text { - Permite revisar el historial que registra la participación de } \\
\text { los estudiantes. }\end{array}$ \\
\hline \multirow{2}{*}{ P量PowerPoint } & - Invita a la síntesis. \\
\hline & - Permite la combinación de texto e imagen. \\
\hline ez। & - Establece un orden para presentaciones orales. \\
\hline \multirow{3}{*}{ w梪 Word } & $\begin{array}{l}\text { - Permite ver la génesis del texto, que no es un proceso } \\
\text { lineal. }\end{array}$ \\
\hline & - Permite insertar comentarios para la revisión. \\
\hline & - Tiene corrector que ayuda a evitar errores. \\
\hline - Blogger & $\begin{array}{l}\text { - Es fácil y sencillo, permite incorporar otros recursos, como } \\
\text { imagen y video. }\end{array}$ \\
\hline \multirow{2}{*}{ (4) WORDPRESS } & - Se escribe para otros y permite leer lo que escriben otros. \\
\hline & - Se publica y el lector es más real. \\
\hline \multirow{2}{*}{ slideshare } & - Permite publicar y compartir materiales. \\
\hline & - Alivia la carga de archivos en el aula virtual. \\
\hline \multirow{3}{*}{ 3) Ewitker } & - Es popular y de fácil acceso. \\
\hline & - Permite la interacción en tiempo real. \\
\hline & - Invita a la síntesis y la creatividad lingüística. \\
\hline
\end{tabular}

Fuente: elaboración propia.

En relación con los entornos de trabajo que tienen a su disposición, coinciden en que el uso de plataformas institucionales, en particular Moodle, no resulta sencillo y en que los estudiantes muestran un cierto rechazo para ingresar y realizar actividades en ese entorno. Es por ello que en muchos casos han optado por proponer actividades de lectura y escritura en redes sociales, especialmente Facebook, por ser ambientes que resultan más familiares para los 
estudiantes. El uso de documentos de Google Drive y blogs para la escritura ha sido otra práctica recurrente entre los entrevistados, así como presentaciones en PowerPoint y procesadores de texto como Word. Algunos se han aventurado al uso de herramientas que desconocían y que decidieron explorar con fines didácticos, como fue el caso de SoundCloud, Audacity y Storybird. Finalmente, los entrevistados que han contado con apoyo institucional o gabinete de expertos en programación han podido diseñar sus propios entornos de trabajo, como aplicaciones para videojuegos y propuestas georreferenciadas, o bien han podido hacer adaptaciones de las plataformas institucionales.

Los obstáculos con los que se han encontrado a la hora de implementar actividades con TIC han sido en su mayoría problemas de infraestructura o cuestiones técnicas asociadas a las limitaciones de las plataformas o entornos utilizados y la falta de conectividad. Comparten también la preocupación por el tiempo adicional que requiere el diseño de actividades de lectura y de escritura con tecnología, así como la puesta en marcha y seguimiento de dichas propuestas.

Para las actividades de lectura, los entrevistados han visto como obstáculo la falta de recursos para diseñar aplicaciones o dispositivos que permitan su reutilización con otros textos, a modo de plantilla que el docente pueda completar con sus textos y preguntas de comprensión. Para las actividades de escritura, un inconveniente recurrente es la falta de recursos humanos para un seguimiento personalizado de las producciones de cada estudiante. En estos dos grandes obstáculos se han inspirado gran parte de las propuestas realizadas por los entrevistados.

Para estos docentes, con la inclusión de las TIC en el trabajo de aula, surgen -o se visibilizan-desafíos. Entre estos mencionaron lograr que los estudiantes dialoguen a través de la escritura, que lleguen a acuerdos, que colaboren de modo genuino o produzcan con otros y que sus trabajos sean visibles para el resto del grupo o para la comunidad. También consideran un desafío el aprendizaje de la escritura sustentada en citas y la reformulación e integración de la palabra del otro en el texto propio. Ante estos retos valoran el uso de las TIC como una posibilidad de disminuir la postergación del inicio de la escritura, acompañar el proceso y tomar distancia para visualizar el camino recorrido. También destacan la facilidad con que las tecnologías permiten contextualizar las lecturas y abrir puentes a otros recursos para enriquecer esos textos.

\subsection{Revisitar la enseñanza de la lectura y la escritura mediada por tecnología}

Las propuestas de enseñanza de la lectura y la escritura mediadas por tecnología, tal como las describieron los docentes entrevistados, dejan ver una 
intención clara de aprovechar las potencialidades de las tecnologías para dar respuesta a muchas de las dificultades con que se encuentran los estudiantes a la hora de abordar la lectura y la escritura de textos en la educación superior. Se trata de una decisión didáctica de revisitar las prácticas tradicionales de lectura y escritura a la luz de las configuraciones actuales.

En este sentido, es posible enumerar las potencialidades de los recursos tecnológicos:

- La posibilidad de enfrentar al estudiante con un lector real, un otro que lee lo que uno escribe, aparece como un rasgo destacado de los procesos estudiados. Ya no es el lector imaginario o la profesora que pidió el texto para leerlo en su casa y hacernos una devolución, ahora puede ser cualquiera, ahora cualquiera puede leer nuestro texto (Clerici, 2017a).

- Segundo, el acompañamiento durante el proceso de escritura, la presencia del docente en cada momento del recorrido, el andamiaje durante el camino (Clerici, 2017b).

- Una tercera potencialidad, asociada a la anterior, es el registro que va quedando de ese camino recorrido y la oportunidad de pararse para ver cada momento del proceso, volver hacia atrás la mirada para ver lo andado. Esto se presenta como una oportunidad tanto para el estudiante como para el profesor (Clerici, 2017b).

- Finalmente, la apertura que genera a otros textos y contextos que enriquecen los procesos de lectura; podemos entrar y salir del texto, podemos hacer una lectura enlazada a múltiples textos, convencionales y no convencionales, que nos ayudan en la comprensión.

Estas son las potencialidades de la tecnología para abordar la enseñanza de la lectura y la escritura desde una preocupación genuina de dar respuesta a las principales dificultades que encuentran los jóvenes que ingresan al nivel superior y se encuentran con nuevos modos de leer y escribir propios del ámbito académico. Estas dificultades de los estudiantes se transforman en un desafío para los docentes. La Tabla 2 ilustra algunas estas dificultades-desafíos y cómo han utilizado la tecnología para hacerles frente. 
Clerici, Carolina I Revisitar la enseñanza de la lectura y la escritura mediada por tecnologías en educación superior

Tabla 2. Algunos usos de las TIC y propuestas de los docentes para hacer frente a las dificultades de los estudiantes para leer y escribir

\begin{tabular}{|c|c|}
\hline $\begin{array}{l}\text { Algunas dificultades de los } \\
\text { estudiantes para leer y } \\
\text { escribir: los desafíos }\end{array}$ & $\begin{array}{l}\text { Algunas alternativas para hacer frente a esos desafíos con } \\
\text { TIC: las resoluciones didácticas }\end{array}$ \\
\hline $\begin{array}{l}\text { No se sienten motivados } \\
\text { para leer }\end{array}$ & $\begin{array}{l}\text { Leer en movimiento, como una búsqueda del tesoro (Do- } \\
\text { cente } 12 \text { ) } \\
\text { Leer como insumo para competir (Docente 13) }\end{array}$ \\
\hline $\begin{array}{l}\text { Se aburren frente a textos } \\
\text { extensos y lineales, no sos- } \\
\text { tienen el esfuerzo }\end{array}$ & $\begin{array}{l}\text { Proponer la estrategia de rompecabezas, cada uno lee una } \\
\text { parte (Docente } 1 \text { y Docente } 2 \text { ) } \\
\text { Leer por partes y en movimiento con una propuesta georre- } \\
\text { ferenciada (Docente 12) } \\
\text { Jugar un videojuego con textos discontinuos (Docente 13) }\end{array}$ \\
\hline $\begin{array}{l}\text { No cuentan con universos de } \\
\text { sentido para comprender lo } \\
\text { que leen }\end{array}$ & $\begin{array}{l}\text { Anticipar términos desconocidos e incluir enlace a defini- } \\
\text { ciones o glosarios (Docente } 2 \text { y Docente } 5 \text { ) } \\
\text { Generar puentes a otros textos (Docente 5) } \\
\text { Usar disparadores que enriquezcan y predispongan a la } \\
\text { lectura (Docente 10) }\end{array}$ \\
\hline $\begin{array}{l}\text { No comprenden las ideas } \\
\text { centrales de un texto }\end{array}$ & $\begin{array}{l}\text { Solicitar palabras clave para elaborar una presentación } \\
\text { oral (Docente 2) } \\
\text { Diseñar cuestionarios con autocorrección para practicar } \\
\text { fuera del aula (Docente 3) } \\
\text { Pasar del texto expositivo a la presentación digital (Docen- } \\
\text { te 10) }\end{array}$ \\
\hline $\begin{array}{l}\text { Posponen el inicio de la es- } \\
\text { critura }\end{array}$ & $\begin{array}{l}\text { Iniciar la escritura en forma lúdica (Docente 6) } \\
\text { Completar Esquemas Digitales de Escritura mientras se lee } \\
\text { (Docente 7) }\end{array}$ \\
\hline $\begin{array}{l}\text { No conocen géneros discur- } \\
\text { sivos, no saben cómo escribir } \\
\text { textos académicos }\end{array}$ & $\begin{array}{l}\text { Escribir en forma colaborativa para ver cómo escriben } \\
\text { otros (Docente } 6 \text { ) } \\
\text { Compartir textos bien resueltos, modelos, ejemplos (Docen- } \\
\text { te } 7 \text { y } 12 \text { ) }\end{array}$ \\
\hline $\begin{array}{l}\text { Copian y pegan textos de } \\
\text { internet, no citan, no toman } \\
\text { conciencia de la referencia a } \\
\text { fuentes }\end{array}$ & $\begin{array}{l}\text { Colorear la palabra del otro para destacar lo propio y lo } \\
\text { ajeno, publicar los escritos (Docente } 1 \text { ) } \\
\text { Buscar información sobre el autor a medida que se lee (Do- } \\
\text { cente 4) } \\
\text { Fichar en un Esquema Digital de Escritura (Docente 7) }\end{array}$ \\
\hline $\begin{array}{l}\text { No revisan el texto o lo ha- } \\
\text { cen de forma superficial }\end{array}$ & $\begin{array}{l}\text { Marcar comentarios en el escrito del estudiante y pedir } \\
\text { reformulación (Docente } 2 \text { ) } \\
\text { Reescribir en nuevos formatos (Docente 6) } \\
\text { Proyectar fragmentos para que vean sus errores (Docente 9) } \\
\text { Publicar lo que se escribe (Docente } 11 \text { y 12) } \\
\text { Escribir en forma colaborativa (Docente 14) }\end{array}$ \\
\hline $\begin{array}{l}\text { No saben ejercitar fuera de } \\
\text { la clase, en especial habili- } \\
\text { dades y no contenido }\end{array}$ & $\begin{array}{l}\text { Diseñar cuestionarios de autocorrección para practicar } \\
\text { fuera del aula (Docente } 3 \text { y Docente 14) }\end{array}$ \\
\hline
\end{tabular}

Fuente: elaboración propia. 
En una lectura transversal de estas propuestas, es posible construir tres categorías de análisis que dan cuenta de revisiones de las formas tradicionales de enseñanza de la lectura y la escritura en clave contemporánea, desde los nuevos modos de leer, escribir y aprender con tecnología, y desde el modo en que lo digital impacta en la comunicación. Se recrean, se reinventan, se revisan estrategias que son significativas para el campo de la alfabetización académica. En este contexto, las estrategias que terminan resolviendo algunas de las dificultades son aquellas que se vinculan con la diversidad de miradas, con el mundo del juego y con la posibilidad de manipulación del texto.

Estas tres categorías que, como construcciones de esta investigación, atraviesan las estrategias de enseñanza que piensan estos docentes a la hora de abordar las dificultades para leer y escribir con que llegan los jóvenes estudiantes al mundo académico nos permiten revisar las maneras en las que los docentes vuelven a dar sentido a sus prácticas en el marco del reconocimiento de lo que pueden ofrecer las tecnologías para potenciar mejores procesos de aprendizaje.

Propuestas habituales que los docentes ponen en juego para abordar la enseñanza de la lectura y la escritura se revisan a la luz de los fenómenos culturales contemporáneos. En este sentido, estos fenómenos tecnológicos, estas prácticas asociadas a la cultura digital son la lente desde la que los docentes revisitan sus estrategias. Algunas de ellas, que son valiosas para el campo y la enseñanza de la lengua, se ven resignificadas en el marco de la cultura digital, las visiones que los estudiantes tienen de ellas, los modos en que se sienten más identificados con estas prácticas, la ludificación, las nuevas formas de mirar y de mirarse.

\section{Discusión y conclusiones}

A lo largo de este trabajo se ha buscado conocer algunas prácticas de enseñanza de la lectura y la escritura mediadas por tecnología en el nivel superior. El foco ha estado en las actividades que proponen los docentes a sus estudiantes, las razones por las cuales deciden utilizar la tecnología en el aula y el modo en que se da la mediación tecnológica para el aprendizaje de la lectura y la escritura para el nivel superior en esas propuestas. Se trata, en su mayoría, de usos modestos de la tecnología, pero relevantes para la enseñanza, como acercamientos a la problemática y como inspiración para continuar en este camino. Se parte de un reconocimiento de cómo las nuevas tecnologías atraviesan las formas en que el conocimiento se construye en la actualidad en todas sus versiones, disciplinares y no disciplinares. En este marco, la idea 
de inclusión y mediación de las tecnologías reconoce estos atravesamientos, busca entenderlos y recuperarlos a la hora de concebir propuestas didácticas (Maggio, 2012).

Esta investigación aborda las maneras en que la inclusión y mediación de las tecnologías transforman la enseñanza. Son «significativas» como una aliadas a la hora de abordar las dificultades que habitualmente encuentran estos docentes para la enseñanza de la lectura y la escritura al encontrarse con estudiantes que no logran sostener la lectura de textos extensos, comprender lo que leen cuando no cuentan con conocimientos previos, escribir cuando no conocen el género discursivo solicitado, iniciar el proceso de escritura, revisar el texto en profundidad, citar fuentes sin incurrir en plagio, etc.

En síntesis, el análisis llevado a cabo de las prácticas de enseñanza en esta investigación pone en evidencia que las principales estrategias de que se valen los entrevistados para abordar las dificultades de los estudiantes para leer y escribir dan cuenta de un docente que se anticipa a ellas y toma de la tecnología su potencial para la enseñanza. Los docentes, en ese reconocimiento, revisan, vuelven a pensar sus estrategias didácticas en la contemporaneidad, las revisitan en el marco de los escenarios sociales, culturales, comunicacionales contemporáneos. En este sentido, la diversidad de miradas, el mundo del juego y la posibilidad de manipulación del texto son rasgos centrales en estas recreaciones docentes.

Esta investigación aborda las maneras en las que un docente que reconoce que la tecnología atraviesa los modos de conocer (Maggio, 2012) y se ubica en un lugar de «incomodidad creativa» para desafiar la propia práctica (Lion, 2012: 39). Se trata de docentes conmovidos por la tecnología (Kap, 2014) que están «convencidos» de que ya no es posible enseñar sin reconocer el atravesamiento de esta en las prácticas de la enseñanza (Maggio, Lion y Perosi, 2014); docentes que buscan «artesanalmente» revisar su práctica (Alliaud, 2017).

Los resultados de este estudio permiten sostener que estos docentes han encontrado un sentido pedagógico y didáctico potente (Maggio, 2012), han logrado reinventar algunas de sus propuestas de enseñanza de la lectura y la escritura, han visto como potencialidad de las nuevas tecnologías el hecho de que hoy podemos enfrentarnos con un lector real, jugar con el texto y entender su complejidad.

Además, en línea con uno de los principales objetivos de esta investigación, la posibilidad de observar el trabajo de colegas y el modo en que piensan sus propuestas de enseñanza de lectura y escritura mediada por tecnología, así como la relataron los entrevistados, desde su propia vivencia, es ineludiblemente una fuente poderosa de inspiración para quienes enfrentamos el 
desafío que nos presenta este campo disciplinar y buscamos revisar el abordaje de la lectura y la escritura en clave didáctica.

Finalmente, la recuperación del valor de la lectura y la escritura no radica solamente en el hecho de ser medios para acumular información o para rendir cuentas del conocimiento aprendido sino, principalmente, como instrumentos poderosos para generar y transformar conocimiento, mejorar la calidad de los aprendizajes e involucrar a los estudiantes en su propio proceso de formación. La lectura y la escritura, en tanto constructoras y reconstructora del sujeto y de la cultura, son habilidades esenciales de las que debe ocuparse la educación; son instrumentos que nos permiten penetrar la cultura y ser penetrados por ella, son la vía de acceso al pasado codificado y al presente que no vemos con nuestra experiencia directa. La formación de lectores críticos y la construcción de la ciudadanía son un propósito central de las instituciones educativas.

El acceso y el dominio pleno de estas habilidades es un derecho y es una vía para posibilitar prácticas más democratizadoras e inclusivas.

\section{Referencias bibliográficas}

AlLIAUD, A. (2017). Los artesanos de la enseñanza: Acerca de la formación de maestros con oficio. Buenos Aires: Paidós.

ÁlVAREZ, G. y Bassa, L. (2013). TIC y aprendizaje colaborativo: el caso de un blog de aula para mejorar las habilidades de escritura de los estudiantes preuniversitarios. Revista de Universidad y Sociedad del Conocimiento, 10(2), 5-19. DOI: http:// dx.doi.org/10.7238/rusc.v10i2.1740

ÁlvareZ, G.; García, M. y Qués, M. E. (2010). Entornos virtuales de aprendizaje y didáctica de la Lengua. Una propuesta para mejorar las habilidades de reformulación productiva de estudiantes preuniversitarios. Revista Q, 9(5), 1-24. Disponible en: http://eav.upb.edu.co/RevQ/ ediciones/12/343/343.pdf

Álvarez, G. y Taboada, M. B. (2016). Propuestas didácticas mediadas por tecnologías digitales para el desarrollo de competencias de lectura y escritura académicas. Revista Guillermo de Ockham, 14(2), 83-91. DOI: 10.21500/22563202.2336

Apolo, D.; Bayés, M. y Hermann, A. (2016). Cambios educativos en los procesos de lectura digital: la pedagogía del ciberespacio como estrategia de procesamiento de contenidos en la era de internet. Redes.com, 12, 222-239. DOI: 10.15213/redes.n12.p222

ARnAo VÁsquez, M. O. y Gamonal Torres, C. E. (2016). Lectura y escritura con recursos TIC en educación superior: Evaluación de la competencia digital. Innoeduca. International Journal of Technology and Educational Innovation, 2(1), 64-73. DOI: 10.20548/innoeduca.2016.v2i1.1046

BUCKINGHAM, D. (2006, marzo 3-4). La educación para los medios en la era de la tec- 
nología digital. Ponencia presentada en el Congreso del décimo aniversario de MED La sapienza di comunicare, Roma, Italia.

BuCKINGHAM, D. (2008). Más allá de la tecnología. Buenos Aires: Manantial.

BURBULES, N. y Callister, T. (2001). Educación: riesgos y promesas de las nuevas tecnologías de la información (Trads. L. Wolfson, A. Oviedo, D. Sagaró, J. Frachia y P. Grosman). Barcelona: Granica.

CARLINO, P. (2003). Leer textos científicos y académicos en la educación superior: Obstáculos y bienvenida a una cultura nueva. Uni-pluri/versidad, 3(2) 17-23.

CASSANY, D. (2002, febrero 18-23). La alfabetización digital. En V. Sánchez Corrales (Ed.), XIII Congreso Internacional de la Asociación de Lingüística y Filología de América Latina (ALFAL), Universidad de Costa Rica, San José de Costa Rica.

CASSANY, D. y Morales, O. A. (2008). Leer y escribir en la universidad: hacia la lectura y la escritura crítica de géneros científicos. Memoralia, 5(2), 69-82.

CASTELLÓ, M. (coord.) (2007). Escribir y comunicarse en contextos científicos y académicos. Barcelona: Graó.

CASTELLS, M. (1999). La era de la información. Economía, sociedad y cultura. La sociedad red. Barcelona: Alianza Editorial.

CLERICI, C. (2017a). La presencia del otro como desafío de la escritura mediada por tecnología. Ensayos, Revista de la Facultad de Educación de Albacete, 32(1), 63-79 DOI: 10.18239/ensayos.v32i1.1285

CLERICI, C. (2017a). La mediación tecnológica en el acompañamiento y la visualización del proceso de escritura. Tendencias Pedagógicas, 30, 227-244.
COPE, B. y Kalantzis, M. (2010). New Media, New Learning. En D. R. Cole y D. L. Pullen (eds.), Multiliteracies in Motion: Current Theory and Practice, 87-104. Nueva York: Routledge.

De Castro, A. E.; Cantillo Oliveros, M. O.; Carbonó Truyó, V. I.; Robles, H. S.; Plaza, D. D.; Guerra Flórez, D.; Rodríguez Fuentes, R. A. y Álvarez, S. (2014). Comprensión Lectora y TIC en la universidad. Apertura. Revista e Innovación Educativa, 6(1), 46-59. Disponible en: http://www.udgvirtual.udg. $\mathrm{mx} /$ apertura/index.php/apertura/article/ view/465/356

De CASTRO, A. E.; Plaza, D. D.; Robles Noriega, H.; Rodríguez Fuentes, R. A.; Cantillo Oliveros, M. O.; Carbonó Truyó, V.; Álvarez Uribe, S. y Guerra Flórez, D. (2015). El efecto del uso de las TIC en la comprensión lectora de español como lengua materna (L1). Revista de Investigación Educativa de la Escuela de Graduados en Educación, 5(10), 43-50. Disponible en: http://rieege.tecvirtual.mx/ index.php/rieege/article/view/158

DUSSEL, I. y Quevedo, L. A. (2010). VI Foro Latinoamericano de Educación; Educación y nuevas tecnologías: los desafíos pedagógicos ante el mundo digital. Buenos Aires: Santillana.

EstienNE, V. M. (2006). Enseñar a leer en la universidad: Una responsabilidad compartida. Revista Científica de UCES, 10(1), 37-46. Disponible en: http://dspace.uces.edu.ar:8180/xmlui/ handle/123456789/322

FIGUeROA, B.; Aillon, M. y Fuentealba, A. (2014). La escritura académica con soporte de esquemas digitales en la formación docente. Revista de Universidad y Sociedad 
del Conocimiento (RUSC), 11(1), 18-32. DOI: 10.7238/rusc.v11i1.1665

FigueroA, B. y Aillon, M. (2015). Escritura académica de un ensayo mediado por el aprendizaje colaborativo virtual. Estudios Pedagógicos, 41(1), 79-91. DOI: 10.4067/ S0718-07052015000100005

FISCHER, H. (2005, abril 27). Educación y cibertecnologías. Conferencia dictada en la Especialización en Educación, Lenguajes y Medios de la Escuela de la Universidad de San Martín, Provincia de Buenos Aires, Argentina. Disponible en: http://www.hervefischer.net/text es.php?idt=11

FUENTEALBA, A.; Figueroa, B. y Aillon, M. (2011). Esquemas Digitales de Escritura (EDE) en Modalidad B-learning para la Alfabetización Académica. Nuevas Ideas en Informática Educativa, Memorias del XVI Congreso Internacional de Informática Educativa, TISE, 7, 88-94. Disponible en: http://www.tise. cl/volumen7/TISE2011/Documento11.pdf

Gallego OrTegA, J. L.; García Guzmán, A. y Rodríguez Fuentes, A. (2013). Cómo planifican las tareas de escritura estudiantes universitarios españoles. Revista Mexicana de Investigación Educativa, 18(57), 599-623.

GLASER, B. y Strauss, A. (1967). The Discovery of Grounded Theory: Strategies for Qualitative Research. Nueva York: Aldine Publishing Company.

GoETZ, J. P. y Le Compte, M. D. (1988). Etnografía y diseño cualitativo en investigación educativa. Madrid: Morata.

GUBER, R. (1991 [2004]). El salvaje metropolitano: Reconstrucción del conocimiento social en el trabajo de campo. Buenos Aires: Paidós. JARAMILlo MARín, P. E. y Ruiz Quiroga, M. (2009). Un caso de integración de TIC que no agrega valor al aprendizaje. Revista Latinoamericana de Ciencias Sociales, Niñez y Juventud, 7(1), 267-287.

JARVIO, A. (2011). La lectura digital en el ámbito de la universidad veracruzana (tesis doctoral). Universidad de Salamanca, Facultad de Traducción y Documentación. Disponible en: http://cdigital.uv.mx/bitstream/123456789/22757/1/Practicas $\% 20$ Lectoras.pdf

JENKINS, H. (2009). Confronting the Challenges of Participatory Culture. Cambridge: The MIT Press.

KAP, M. (2014). Conmovidos por la tecnología. Buenos Aires: Prometeo.

LION, C. (2012). Pensar en red. Metáforas y escenarios. En M. Narodoski y A. Scialabba (comps.). ¿Cómo serán? El futuro de la escuela y las nuevas tecnologías, 29-45. Buenos Aires: Prometeo Libros.

LITWIN, E. (2008). El oficio del docente y las nuevas tecnologías: herramientas, apremios y experticias. Educação Unisinos, 12(3), 167-173. DOI: 10.4013/ edu.20083.01

LÓPEZ-ANDRADA, C. (2016). Discursos en torno a la alfabetización digital y académica: hacia un modelo integrador. Virtualidad, Educación y Ciencia, 12(7), 143-153.

MAGGIO, M. (2012). Enriquecer la enseñanza. Buenos Aires: Paidós.

MAGGIO, M.; Lion, C. y Perosi, V. (2014). Las prácticas de la enseñanza recreadas en los escenarios de alta disposición tecnológica. Polifonías Revista de Educación, 3(5), 101 127. Disponible en: http://www.polifoniasrevista.unlu.edu.ar/sites/www.polifoniasrevista.unlu.edu.ar/files/site/POLIFONIAS\%20 N\%C2\%B0\%205\%20Sept-Oct.\%202014.pdf 
Monereo, C. y Pozo, J. I. (2007). Competencias para (con)vivir con el siglo XXI. Cuadernos de Pedagogía, 370, 12-18.

NAVARRO, F.; Ávila Reyes, N.; Tapia Ladino, M.; Cristovão, V.; Moritz, M. E.; Narváez Cardona, E. y Bazerman, C. (2016). Panorama histórico y contrastivo de los estudios sobre lectura y escritura en educación superior publicados en América Latina. Revista Signos. Estudios de Lingüística, 49(S1), 100-126. DOI: 10.4067/S0718-09342016000400006

OREALC/UNESCO (2013). Enfoque estratégico sobre las TICS en educación en América Latina y el Caribe. Santiago de Chile: UNESCO.

PARODI, G. y Burdiles, G. (eds.). (2015). Leer y escribir en contextos académicos y profesionales: géneros, corpus y métodos. Santiago de Chile: Ariel.

REALE, A. (2008). «Sabía que me gustaba escribir pero nunca pensé que fuera para tanto». Sobre la eficacia de bitácoras y diarios de escritor en el Taller de Expresión I. Jornadas Académicas 2008 «Producir teoría, pensar las prácticas». Buenos Aires: Universidad de Buenos Aires.

Romo GonZÁlez, A. E. y Villalobos Alonzo, M. A. (2009). Comprensión lectora en texto impreso y digital: sus diferencias. En $X$ Congreso Nacional de Investigación Educativa, Consejo Mexicano de Investigación Educativa, México. Disponible en: http://www.comie.org. $\mathrm{mx} /$ congreso/memoriaelectronica/v10/pdf/ area tematica 07/ponencias/0905-F.pdf

SASSEN, S. (2014). Expulsions: Brutality and Complexity in the Global Economy. Cambridge: Harvard University Press.

StRAuss, A. y Corbin, J. (2002). Bases de la investigación cualitativa. (E. Zimmerman trad.). Antioquia: Editorial Universidad de Antioquia.

TAYLOR, S. y Bogdan, R. (1987). Introducción a los métodos cualitativos en investigación. Barcelona: Paidós.

TRILLOS-PACHECO, J. J. (2013). La lectura hipermedial y su incidencia en la comprensión lectora en estudiantes universitarios. Palabra Clave, 16(3), 944-992. DOI: 10.5294/ pacla.2013.16.3.10

VAN DIJCK, J. (2013). The Culture of Connectivity: A Critical History of Social Media. Nueva York: Oxford University Press.

VAN DIJCK, J. (2016). La cultura de la conectividad: Una historia crítica de las redes sociales. (H. Salas trad.). Buenos Aires: Siglo XXI. 